\title{
Direct structural evidence supporting a revolving mechanism in DNA packaging motors
}

\author{
Yao-Gen Shu ${ }^{1 凶}$, Xiaolin Cheng ${ }^{2}$ \\ ${ }^{1}$ Wenzhou Institute, University of Chinese Academy of Sciences, Wenzhou 325001, Zhejiang, China \\ 2 College of Pharmacy, Biophysics Graduate Program, Translational Data Analytics Institute, The Ohio State \\ University, Columbus, OH 43210, USA
}

Received: 15 June 2020 / Accepted: 30 June 2020 / Published online: 4 August 2020

Viruses have evolved two strategies to package genome: either assembling a capsid around their genomes or packaging the genomes into a preformed capsid with packaging motors. Most of the double-stranded (ds) DNA viruses (e.g., tailed bacteriophages and herpesviruses) and dsRNA viruses (e.g., $\phi 6$ and $\phi 12$ bacteriophages) take the latter strategy. The packaging motors are usually called the "terminase" complex, which, in bacteriophages comprises two proteins, termed large (TerL) and small (TerS) terminases (Guo et al. 1987b), while in herpesviruses contains three components-pUL15, pUL28 and pUL33. The unique $\$ 29$ motor complex lacks a TerS but instead contains a 174-nt prohead RNA (pRNA) (Guo et al. 1987a). However, it has long been debated whether the viral genome packaging motors operate via a rotatory or revolving mechanism and what is their oligomeric state in action-a hexamer or a pentamer? And now, published in a recent issue of Protein \& Cell, Yang et al. have hopefully ended these 20-year long fervent debates by determining high-resolution structures of the herpesvirus terminase complex to reveal the six-fold symmetry with a potential revolving mechanism (Yang et al. 2020).

Molecular motors are enzymes that convert a chemical scalar (ATP) into a physical vector (unidirectional motion). They are also called nanomachines as they are all at the nanoscale. Molecular motor is a mesoscopic prototype of a non-equilibrium system of "soft matter + ATP”. Unlike linear motor kinesin, which consumes one ATP molecule for each $8 \mathrm{~nm}$ step with a hand-over-hand mechanism (Li et al. 2018), and reversible rotary motor $\mathrm{F}_{\mathrm{o}} \mathrm{F}_{1}$-ATPase, which synthesizes/

$\bowtie$ Correspondence: shuyaogen@wiucas.ac.cn (Y.-G. Shu) hydrolyzes one ATP molecule for each $120^{\circ}$ step with a binding-change-mechanism (Shu and Lai 2008), the framework of the genome packaging motors remains elusive. Although rotational motion with tight mechanochemical coupling was long thought as a common mechanism for the DNA packaging motors (Hendrix 1998; Serwer 2003; Simpson et al. 2000), a revolving framework of DNA translocation has recently been proposed for the $\$ 29$ dsDNA packaging motor (Guo et al. 2019; Schwartz et al. 2013; Zhao et al. 2016).

The oligomeric state of the DNA packaging motors has also been debated for decades. The debate initiated in bacteriophage $\$ 29$ where low-resolution cryo-EM reconstructions slightly favored fivefold symmetry averaged structures (Morais et al. 2001, 2008; Simpson et al. 2000), whereas a large body of biochemical data supported a hexameric ring structure (Guo et al. 1998; Ibarra et al. 2000; Schwartz et al. 2013; Trottier and Guo 1997). The same mystery carries over to bacteriophage T4 where atomic structures of the motor proteins seemed able to fit into both 5- and sixfold symmetry averaged low-resolution ( $>30 \AA$ A $)$ cryo-EM densities (Sun et al. 2008). All these controversies on the composition, architecture, and packaging mechanism are rooted from the lack of a high-resolution threedimensional structure of the viral packaging motors.

Recently, Yang et al. have determined the first atomic structures of a herpesvirus terminase complex in both apo and ATP mimic-bound states (Yang et al. 2020). When the three components of the herpesvirus terminase complex-the ATPase/terminase pUL15 and two regulator/fixer proteins, pUL28 and pUL33-were coexpressed using a baculovirus-based expression system, the terminase complex assembled predominantly into a hexameric ring in vitro. Each subunit of the hexameric 
ring is a heterotrimer formed by the three proteins (pUL15, pUL28 and pUL33) interdigitating with each other. The terminase pUL15 folds into an "L" shaped structure, containing five functional domains: N-lasso (residues 1-152), strut (residues 153-252), ATPase (residues 253-413), regulator (residues 414-478) and nuclease (residues 479-735). Structural analysis also ambiguously identified R346 as the trans-acting arginine finger that extends from an ATPase subunit to its adjacent ATP binding pocket to interact with the $\gamma$ phosphate.

Although atomic structures of several full-length viral large terminase subunits, such as T4 gp17 (Sun et al. 2008) and Sf6 gp2 (Zhao et al. 2013), are available, herpesvirus pUL15 is the only TerL structure determined in a potentially "functional" oligomeric state. Therefore, the pUL15 structure provides an important template for understanding the relative orientation of the ATPase motors with respect to their DNA substrates and how the individual subunits interact with each other in their oligomeric state. We performed simple structural superposition of the three monomeric TerL structures using their ATPase domains as a reference. We focused on the three domains common to most DNA packaging motors, the ATPase domain (corresponding to the N-terminal domain of $\phi 29$ gp16, referred to here as NTD), the regulator domain (corresponding to the linker domain of $\phi 29 \mathrm{gp} 16$ ) and the nuclease domain (corresponding to the C-terminal domain of $\phi 29$ gp16, referred to here as CTD). As shown in Fig. 1, despite their distant relation in the viral family, all the three ATPase domains are aligned remarkably well while large variations are observed in the spatial arrangements of individual domains. Both the NTD-CTD separation and the orientation of CTD relative to NTD vary among the three TerLs. In herpesvirus pUL15, NTD and CTD make no direct contact with each other while both T4 gp17 and Sf6 gp2 show extensive interactions between the two domains. Another intriguing observation is that the catalytic site of the nuclease domain in pUL15 opens towards the adjacent subunit instead of DNA, suggesting the nuclease activity is inhibited during translocation (Yang et al. 2020). This information is, however, not immediately clear from the monomeric structure of either T4 gp17 or Sf6 gp2, but based on the aligned structures, it is evident that both nuclease sites are inaccessible to DNA, and conformational changes would be required for both $\mathrm{T} 4 \mathrm{gp} 17$ and Sf6 gp2 to expose their nuclease sites for DNA cleavage upon packaging.

Furthermore, these results lend structural support to the revolving mechanism rather than the rotatory mechanism of dsDNA translocation. The six ATPase domains of pUL15 form a central channel with conserved basic patches conducive to DNA binding. A diameter of $39 \AA$ for the central channel, in line with that of the herpesvirus portal ( $\sim 36 \AA$ in diameter), is much wider than the size of a B-form dsDNA ( $\sim 20 \AA$ in diameter), which clearly favors the revolving model, since if it were a rotatory motor, a channel smaller than $20 \AA$ in diameter should have been detected because close contact between the channel and the substrate is necessary for a rotational motion to occur. These findings are also supported by molecular dynamics simulations of the $\phi 29$ connector that revealed a quite heterogeneous distribution of stiff and soft regions of the motor channel, compatible with the one-way revolution model (Kumar and Grubmüller 2014). Interestingly, using building blocks resolved in the hexameric structures, a hypothetical pentameric ring structure could be created to maintain reasonable contact between adjacent subunits, but such a model with much narrower constrictions (19-24 A) would exclude the revolving translocation mechanism (Yang et al. 2020). Taken together, these new structures provide compelling evidence that the hexameric terminase complex uses a revolving mechanism to translocate dsDNA.

Tremendous efforts have been made to determine three-dimensional structures of individual viral proteins and whole herpes virions (Dai and Zhou 2018; Wang et al. 2018; Yuan et al. 2018), but the resolution was too low to allow meaningful reconstruction of the terminase complex structure. This is partially attributable to the small size of this complex compared to the capsid protein layer as well as the technical difficulties associated with resolving non-icosahedral components in a large icosahedral virus. On the other hand, isolation of an intact terminase complex has been proven extremely challenging. In this study, the authors were able to assemble a hexameric terminase complex in vitro, and cryo-EM of the assembled hexamers in combination with a block-reconstruction method (Yuan et al. 2018; Zhu et al. 2018) has finally led to the first atomic structures of the herpesvirus terminase complex.

Overall, these new atomic structures of the herpesvirus terminase complex provide a reconciling structural view of viral genome packaging in dsDNA viruses. Nevertheless, the detailed revolving mechanism of this dsDNA packaging motor is just beginning to be elucidated because the kinetics of its packaging has not yet been studied quantitatively with a phenomenological model, and many questions related to the kinetic details of the mechanism remain. For example, is the motor a processive motor? Is its mechanochemical coupling tight? Does the revolution include stepping? What is the length of the step? And so on. On the other 
A

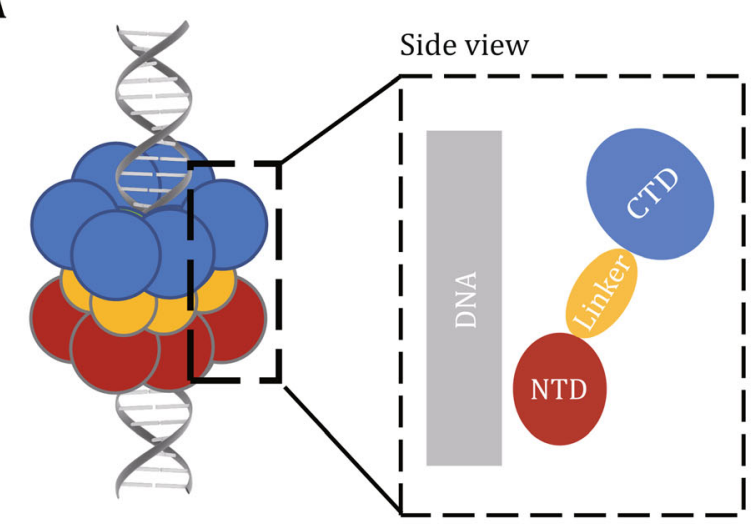

$90^{\circ}$

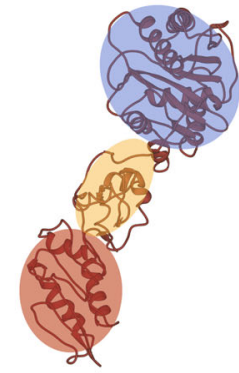

HSV-1

(pUL15)

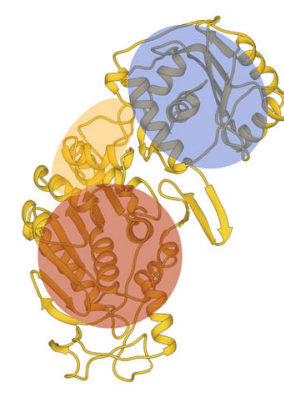

$\mathrm{T} 4$

(gp17)

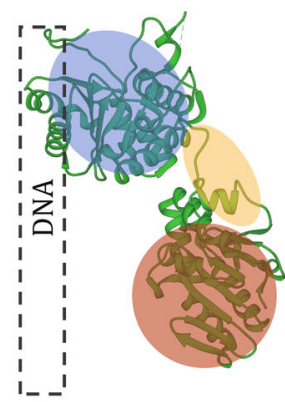

Sf6

(gp2)
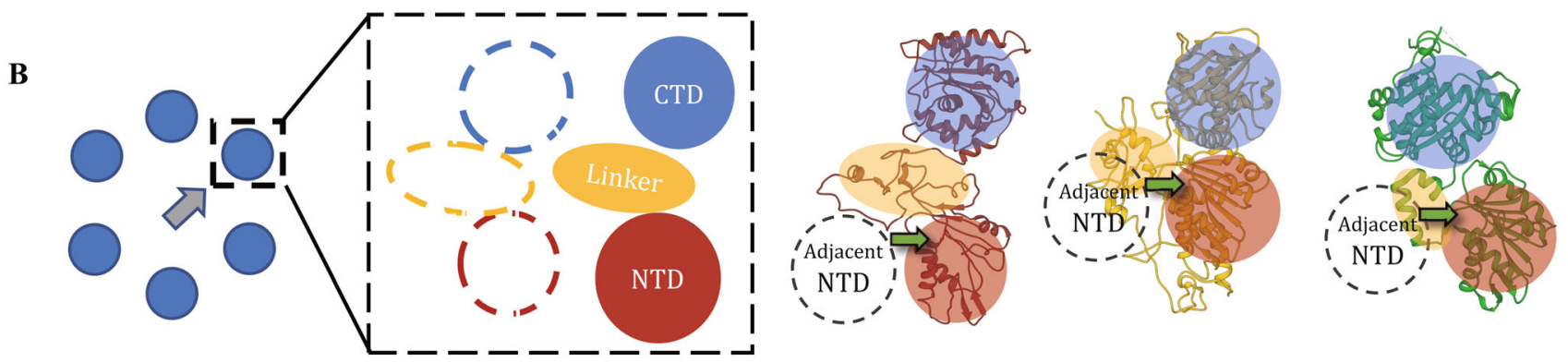

Fig. 1 Comparison of viral packaging motor proteins from the side view (A) and the interior view (B). In the schematic, NTD (N-terminal domain), linker domain and CTD (C-terminal domain) were colored in red, yellow and blue, respectively. The interior view is also denoted by a gray arrow, indicating viewing from the interior of the channel. The green arrows indicate the location of the arginine finger between two neighboring NTDs

hand, the entropy of the genome is changing during packaging, so that the reactive force on the packaging motor is no longer constant. Kinetically, the steady state, which is indispensable for quantitative investigation, disappears.

Acknowledgements X.C. acknowledges the start-up fund from the College of Pharmacy and the Discovery Themes at The Ohio State University. Y-G. Shu acknowledges the financial support by the CAS Strategic Priority Research Program (XDA17010504), National Natural Science Foundation of China (11774358), Key Research Program of Frontier Sciences of CAS (Y7Y1472Y61), and WIUCASYJ2020004.

\section{Compliance with Ethical Standards}

Conflict of interest Yao-Gen Shu and Xiaolin Cheng declare that they have no conflict of interest.

Human and animal rights and informed consent This article does not contain any studies with human or animal subjects performed by any of the authors.

Open Access This article is licensed under a Creative Commons Attribution 4.0 International License, which permits use, sharing, adaptation, distribution and reproduction in any medium or format, as long as you give appropriate credit to the original author(s) and the source, provide a link to the Creative Commons licence, and indicate if changes were made. The images or other third party material in this article are included in the article's Creative Commons licence, unless indicated otherwise in a credit line to the material. If material is not included in the article's Creative Commons licence and your intended use is not permitted by statutory regulation or exceeds the permitted use, you will need to obtain permission directly from the copyright holder. To view a copy of this licence, visit http://creativecommons.org/ licenses/by/4.0/.

\section{References}

Dai X, Zhou ZH (2018) Structure of the herpes simplex virus 1 capsid with associated tegument protein complexes. Science 360(6384):eaa07298. https://doi.org/10.1126/science. aao7298

Guo P, Erickson S, Anderson D (1987a) A small viral RNA is required for in vitro packaging of bacteriophage phi 29 DNA. Science 236(4802):690-694

Guo P, Peterson C, Anderson D (1987b) Prohead and DNA-gp3dependent ATPase activity of the DNA packaging protein gp16 of bacteriophage $\varphi 29$. J Mol Biol 197(2):229-236

Guo P, Zhang C, Chen C, Garver K, Trottier M (1998) Inter-RNA interaction of phage $\varphi 29$ pRNA to form a hexameric complex for viral DNA transportation. Mol Cell 2(1):149-155 
Guo P, Driver D, Zhao Z, Zheng Z, Chan C, Cheng X (2019) Controlling the revolving and rotating motion direction of asymmetric hexameric nanomotor by arginine finger and channel chirality. ACS Nano 13(6):6207-6223

Hendrix RW (1998) Bacteriophage DNA packaging: RNA gears in a DNA transport machine. Cell 94(2):147-150

Ibarra B, Castón JR, Llorca O, Valle M, Valpuesta JM, Carrascosa JL (2000) Topology of the components of the DNA packaging machinery in the phage $\varphi 29$ prohead. J Mol Biol 298(5):807-815

Kumar R, Grubmüller H (2014) Elastic properties and heterogeneous stiffness of the phi29 motor connector channel. Biophys J 106(6):1338-1348

Li M, Ou-Yang Z-C, Shu Y-G (2018) Advances in the study of the mechanochemical coupling of kinesin. Int J Mod Phys B 32(18):1840001.

https://doi.org/10.1142/ S0217979218400015

Morais MC, Tao Y, Olson NH, Grimes S, Jardine PJ, Anderson DL, Baker TS, Rossmann MG (2001) Cryoelectron-microscopy image reconstruction of symmetry mismatches in bacteriophage $\varphi 29$. J Struct Biol 135(1):38-46

Morais MC, Koti JS, Bowman VD, Reyes-Aldrete E, Anderson DL, Rossmann MG (2008) Defining molecular and domain boundaries in the bacteriophage $\$ 29$ DNA packaging motor. Structure 16(8):1267-1274

Schwartz C, De Donatis GM, Zhang H, Fang H, Guo P (2013) Revolution rather than rotation of AAA+ hexameric phi29 nanomotor for viral dsDNA packaging without coiling. Virology 443(1):28-39

Serwer P (2003) Models of bacteriophage DNA packaging motors. J Struct Biol 141(3):179-188

Shu Y-G, Lai P-Y (2008) Systematic kinetics study of FoF1-ATPase: analytic results and comparison with experiments. J Phys Chem B 112(42):13453-13459
Simpson AA, Tao Y, Leiman PG, Badasso MO, He Y, Jardine PJ, Olson $\mathrm{NH}$, Morais MC, Grimes S, Anderson DL (2000) Structure of the bacteriophage $\varphi 29$ DNA packaging motor. Nature 408(6813):745-750

Sun S, Kondabagil K, Draper B, Alam TI, Bowman VD, Zhang Z, Hegde S, Fokine A, Rossmann MG, Rao VB (2008) The structure of the phage T4 DNA packaging motor suggests a mechanism dependent on electrostatic forces. Cell 135(7):1251-1262

Trottier M, Guo P (1997) Approaches to determine stoichiometry of viral assembly components. J Virol 71(1):487-494

Wang J, Yuan S, Zhu D, Tang H, Wang N, Chen W, Gao Q Li Y, Wang J, Liu H (2018) Structure of the herpes simplex virus type 2 C-capsid with capsid-vertex-specific component. Nat Commun 9(1):1-10

Yang Y, Yang P, Wang N, Chen Z, Su D, Zhou ZH, Rao Z, Wang X (2020) Architecture of the herpesvirus genome-packaging complex and implications for DNA translocation. Protein Cell 11(5):339-351

Yuan S, Wang J, Zhu D, Wang N, Gao Q Chen W, Tang H, Wang J, Zhang X, Liu H (2018) Cryo-EM structure of a herpesvirus capsid at $3.1 \AA \AA$. Science 360(6384):eaao7283. https://doi. org/10.1126/science.aao7283

Zhao H, Christensen TE, Kamau YN, Tang L (2013) Structures of the phage Sf6 large terminase provide new insights into DNA translocation and cleavage. Proc Natl Acad Sci USA 110(20):8075-8080

Zhao Z, De-Donatis GM, Schwartz C, Fang H, Li J, Guo P (2016) An arginine finger regulates the sequential action of asymmetrical hexameric ATPase in the double-stranded DNA translocation motor. Mol Cell Biol 36(19):2514-2523

Zhu D, Wang X, Fang Q, Van Etten JL, Rossmann MG, Rao Z, Zhang X (2018) Pushing the resolution limit by correcting the Ewald sphere effect in single-particle Cryo-EM reconstructions. Nat Commun 9(1):1-7 